\title{
Giant Magnetoresistance Effect in Ni-Fe-Mo Alloys
}

\author{
Shih-Fong Lee ${ }^{1, \mathrm{a}}$ and Jung-Chuan Fan ${ }^{1, \mathrm{~b}}$ \\ ${ }^{1}$ Department of Electrical Engineering, Da-Yeh University, Changhua, Taiwan 51591 \\ sflee@mail.dyu.edu.tw ${ }^{1},{ }^{b}$ fanjc@mail.dyu.edu.tw
}

\begin{abstract}
The properties of ferroelectricity and ferromagnetic in the magnetic materials have been extensively discussed recently. These magnetic materials can be used to fabricate novel microelectronic devices. In this study, the electrical properties of the magnetic materials consisting of nickel $(\mathrm{Ni})$, iron $(\mathrm{Fe})$ and molybdenum $(\mathrm{Mo})$ were investigated. The chemical compositions of these Ni-Fe-Mo magnetic materials were measured with energy diffraction spectroscopy (EDS), while their crystalline structures were determined from X-ray diffraction (XRD). Magnetic shielding has been observed in the magnetic materials sintered at temperatures $400^{\circ} \mathrm{C}$ and $600^{\circ} \mathrm{C}$. Both $\mathrm{FeNi}_{3}$ and MoNi crystal structures were found in the magnetic materials as determined from X-ray diffraction (XRD). In addition, giant magnetic resistance (GMR) effect was observed in the low magnetic field in these magnetic materials. The MR ratio is about $15 \%$ in the magnetic material sintered at $600^{\circ} \mathrm{C}$. It reveals that both $\mathrm{FeNi}_{3}$ and MoNi crystal structures contribute to the giant magnetic resistance (MR) in these magnetic materials.
\end{abstract}

\section{Introduction}

Magnetoresistance (MR) is the property that the electrical resistance of a material can be modified by an external magnetic field. Ordinary magnetoresistance (OMR) effect was first discovered by William Thompson (Lord Kevin) in 1856.[1] He found that the resistance increases when the current is in the same direction as the magnetic force and decreases when the current is at $90^{\circ}$ to the magnetic force. Ordinary magnetoresistance (OMR) can vary only 5 percent and has no relation to the magnetic materials used.

On the other hand, anisotropic magnetoresistance (AMR) has a dependence of electrical resistance on the angle between the direction of electric current and direction of magnetization. [2] The effect arises from the simultaneous action of magnetization and spin-orbit interaction and its detailed mechanism depends on the material. AMR of new materials is being investigated and variation in resistance up to $50 \%$ have been observed in some ferromagnetic uranium compounds.[3-5]

\footnotetext{
* Corresponding author: authorsflee@mail.dyu.edu.tw
} 


\section{Experimental Details}

In this study, the magnetic material under study is a compound composed of nickel (Ni), iron (Fe) and molybdenum (Mo). Ni-Fe alloy is a permeable alloy with a high magnetic permeability, low coercivity, near zero magnetostriction, and significant anisotropic magnetoresistance. The low magnetostriction is critical for industrial applications, where variable stresses in thin films would otherwise cause a large variation in magnetic properties. Depending on the strength and direction of applied magnetic field, the electrical resistivity of $\mathrm{Ni}-\mathrm{Fe}$ alloy may vary up to $5 \%$. Ni-Fe alloys typically have the face centered cubic (fcc) crystal structure with a lattice constant of approximately $0.355 \mathrm{~nm}$ in the vicinity of a nickel concentration of $80 \%$. Ni-Fe alloy is used in transformer laminations and magnetic recording head sensors.

With an addition of 5 percent molybdenum, the resulting Ni-Fe-Mo alloys have even higher permeability. Ni-Fe-Mo alloy is a magnetically soft material. The resistivity of Ni-Fe-Mo alloy is $0.6 \Omega \cdot \mathrm{mm}^{2} / \mathrm{m}$. It has an extremely high magnetic permeability and a low coercivity. Ni-Fe-Mo alloy is used in manufacturing of components in radio engineering, telephony, and telemechanics instruments.

The Ni-Fe-Mo alloy samples were prepared by powder metallurgy. The press and sinter process consists of three basic steps: powder blending (pulverisation), die compaction, and sintering. First, $\mathrm{Ni}(8 \mathrm{~g}), \mathrm{Fe}(1.5 \mathrm{~g})$, and $\mathrm{Mo}(0.42 \mathrm{~g})$ were first ground into fine powders in a mortar, and then hard pressed into mold. Metal powder compaction was carried out at a pressure of $40 \mathrm{~kg} / \mathrm{cm}^{2}$ for 30 minutes. Subsequently, the Ni-Fe-Mo samples were sintered at temperatures $400^{\circ} \mathrm{C}$ and $600^{\circ} \mathrm{C}$ for 8 hours in a high-temperature oven, respectively.

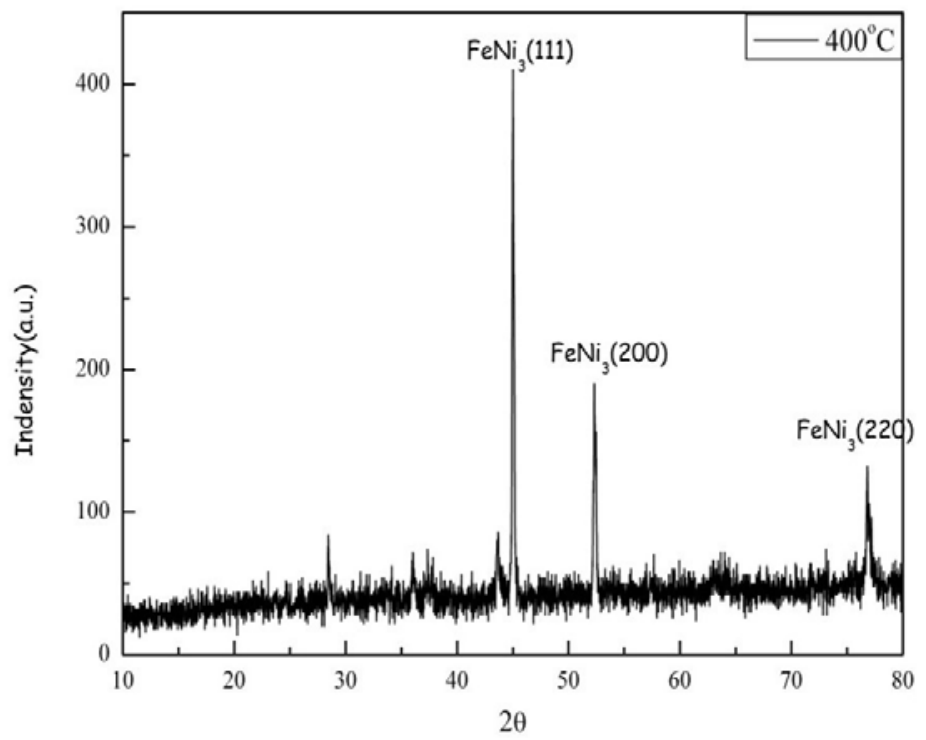

Fig. 1 The XRD spectrum for the Ni-Fe-Mo alloy sintered at $400^{\circ} \mathrm{C}$ in which $\mathrm{FeNi}_{3}$ crystalline structure can be clearly observed. 


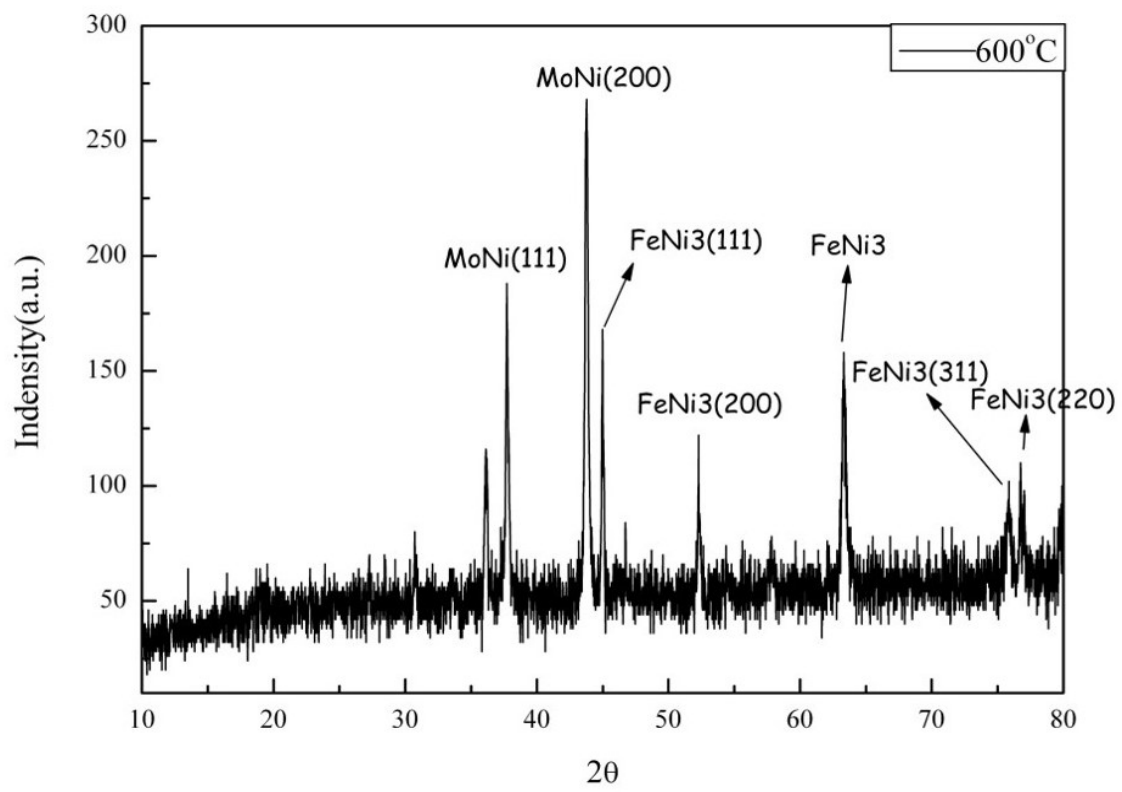

Fig. 2 The XRD spectrum for the Ni-Fe-Mo alloy sintered at $600^{\circ} \mathrm{C}$ in which both $\mathrm{FeNi}_{3}$ and $\mathrm{MoNi}$ crystalline structures can be unambiguously identified.

The chemical compositions of these Ni-Fe-Mo magnetic materials were measured with energy diffraction spectroscopy (EDS), while their crystalline structures were determined from X-ray diffraction (XRD). During the magnetic shielding measurements, the field probe was mounted in between two adjacent Ni-Fe-Mo magnetic material samples. Magnetic field strength was then measured with a Gauss meter by varying the vertical position of the probe to study any variation of magnetic field caused by these Ni-Fe-Mo magnetic materials.

\section{Results and Discussion}

Fig. 1 shows the chemical compositions of Ni-Fe-Mo alloy as determined from EDS. In this figure, background signal corresponding to carbon and oxygen gases during the measurement should be deleted and re-normalized to obtain the weight percentage and atomic percentage of $\mathrm{Ni}, \mathrm{Fe}$, and $\mathrm{Mo}$.

Fig. 2 and Fig. 3 show the XRD spectra derived for the Ni-Fe-Mo alloys sintered at $400^{\circ} \mathrm{C}$ and $600^{\circ} \mathrm{C}$, respectively. In Fig. 2, three characteristic peaks associated with $\mathrm{FeNi}_{3}$ crystalline structure can be clearly observed. In contrast, both $\mathrm{FeNi}_{3}$ and $\mathrm{MoNi}$ crystalline structures can be unambiguously identified for the Ni-Fe-Mo alloy sintered at $600^{\circ} \mathrm{C}$ in the XRD spectrum as shown in Fig. 3. It is also noted that the characteristic peaks associated with MoNi crystalline structure are higher than those associated with $\mathrm{FeNi}_{3}$. Hence, it is reasonable to deduce that $\mathrm{MoNi}$ is the dominant crystalline structure in the Ni-Fe-Mo alloy sintered at $600^{\circ} \mathrm{C}$. It is clear that the formation of MoNi crystalline structure is possible only at an elevated temperature higher than $400^{\circ} \mathrm{C}$. At $600^{\circ} \mathrm{C}$, MoNi tends to be the preferred crystalline structure and most of the $\mathrm{FeNi}_{3}$ crystalline structure have been transformed and recrystallized into MoNi crystalline structure.

Magnetic shielding has been observed for both Ni-Fe-Mo alloy samples sintered at temperatures $400^{\circ} \mathrm{C}$ and $600^{\circ} \mathrm{C}$. During the measurement, the probe was placed in between 
two adjacent NI-Fe-Mo samples and the magnetic field was measured at different positions along the vertical line as shown in Fig. 3. In Fig. 3, a decrease of $0.17 \mathrm{~T}$ in magnetic field was observed for the sample sintered at $400^{\circ} \mathrm{C}$ and a decrease of $0.23 \mathrm{~T}$ was found for the sample sintered at $600^{\circ} \mathrm{C}$. This magnetic shielding can be attributed to the $\mathrm{FeNi}_{3}$ and $\mathrm{MoNi}$ crystalline structures embedded in Ni-Fe-Mo alloys. It seems that MoNi crystalline structure tends to exhibit stronger magnetic shielding than $\mathrm{FeNi}_{3}$ crystalline structure.

$\mathrm{I}-\mathrm{V}$ measurements on these Ni-Fe-Mo alloy samples reveals that the sample sintered at $400^{\circ} \mathrm{C}$ exhibits good I-V characteristics, while the sample sintered at $600^{\circ} \mathrm{C}$ has poor I-V characteristics. In addition, giant magnetoresistance (GMR) effect was observed in the low magnetic field in these magnetic materials. Fig. 4 shows the measured electrical resistance in the presence of uniform magnetic field plotted as a function of applied magnetic field for the $\mathrm{Ni}-\mathrm{Fe}-\mathrm{Mo}$ alloy sintered at $600^{\circ} \mathrm{C}$. The reduction of resistance (MR ratio) is around $15 \%$ in the Ni-Fe-Mo alloy sample sintered at $600^{\circ} \mathrm{C}$, and around $3 \%$ in that sintered at $400^{\circ} \mathrm{C}$. It reveals that both $\mathrm{FeNi}_{3}$ and $\mathrm{MoNi}$ crystal structures contribute to the giant magnetoresistance in these Ni-Fe-Mo magnetic materials. However, MoNi structure seems to have a more pronounced effect on giant magnetoresistance than $\mathrm{FeNi}_{3}$ crystalline structure.

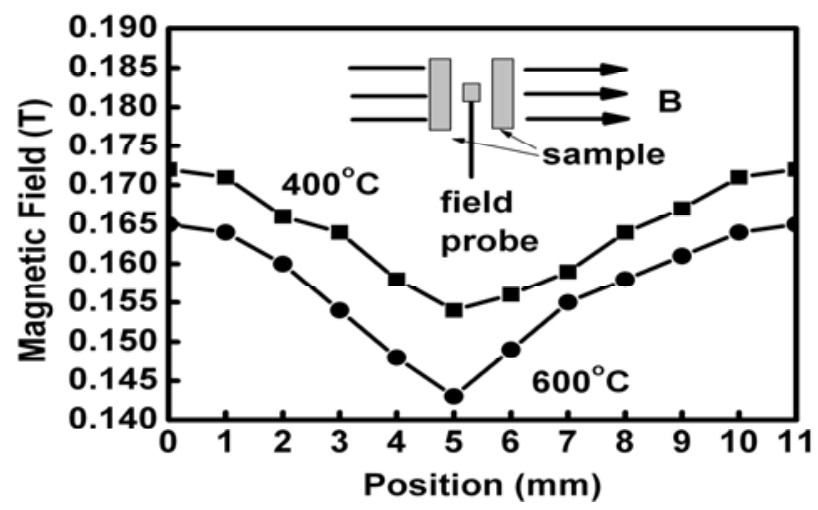

Fig. 3 Magnetic shielding effect observed for the Ni-Fe-Mo alloy samples sintered at temperatures $400^{\circ} \mathrm{C}$ and $600^{\circ} \mathrm{C}$, respectively.

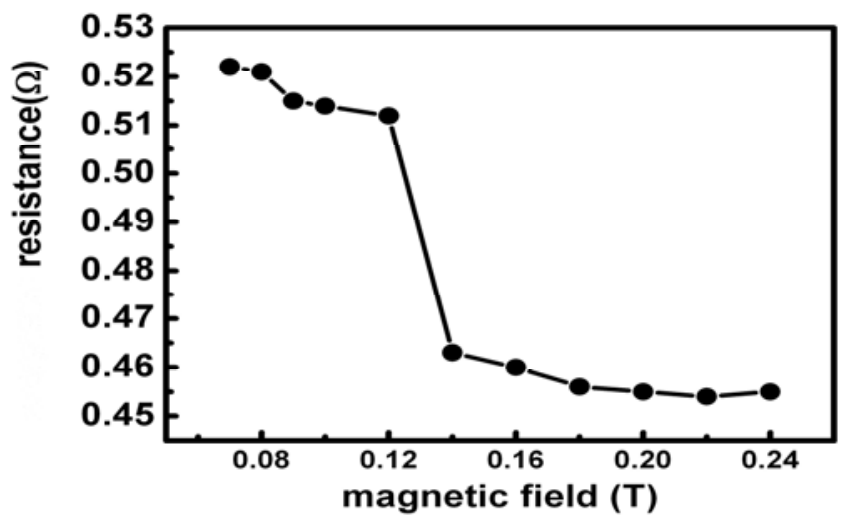

Fig. 4 The measured electrical resistance in the presence of uniform magnetic field plotted as a function of applied magnetic field for the Ni-Fe-Mo alloy sintered at $600^{\circ} \mathrm{C}$. 


\section{Conclusions}

Only $\mathrm{FeNi}_{3}$ crystalline structure was observed in the XRD spectrum for the Ni-Fe-Mo alloy sintered at $400^{\circ} \mathrm{C}$. On the other hand, both $\mathrm{FeNi}_{3}$ and $\mathrm{MoNi}$ crystalline structures has been unambiguously identified in the XRD spectrum for the Ni-Fe-Mo alloy sintered at $600^{\circ} \mathrm{C}$. Thus, the formation of MoNi is possible only at an elevated temperature higher than $400^{\circ} \mathrm{C}$.

Magnetic shielding has been observed in both $\mathrm{Ni}-\mathrm{Fe}-\mathrm{Mo}$ magnetic materials sintered at temperatures $400^{\circ} \mathrm{C}$ and $600^{\circ} \mathrm{C}$. In addition, giant magnetoresistance (GMR) effect was observed in the low magnetic field in these $\mathrm{Ni}-\mathrm{Fe}-\mathrm{Mo}$ magnetic materials. It is concluded that both $\mathrm{FeNi}_{3}$ and $\mathrm{MoNi}$ crystalline structures contribute to the magnetic shielding and giant magnetoresistance in these Ni-Fe-Mo alloys. However, MoNi crystalline structure seems to have a more pronounced effect on magnetic shielding as well as giant magnetoresistance than $\mathrm{FeNi}_{3}$ structure does. With MoNi crystalline structure, the Ni-Fe-Mo alloy sintered at $600^{\circ} \mathrm{C}$ exhibit stronger magnetic shielding $(0.23 \mathrm{~T})$ and larger giant magnetoresistance $(\mathrm{MR}$ ratio $=$ $15 \%)$.

\section{References}

1. A.B. Pippard, Magnetoresistance in Metals, Cambridge University Press, 1989.

2. T. McGuire, R. Potter, Anisotropic magnetoresistance in ferromagnetic $3 \mathrm{~d}$ alloys, IEEE Trans. Magnet. 11(4) (1975) 1018-1038.

3. H.X. Tang, R.K. Kawakami, D.D. Awschalom, M.L. Roukes, Giant planar Hall effect in epitaxial (Ga,Mn)As Devices, Phys. Rev. Lett. 90(10) (2003) 107201.

4. P. Wiśniewski, Giant anisotropic magnetoresistance and magnetothermopower in cubic 3:4 uranium pnictides, Appl. Phys. Lett. 90 (2007) 192106.

5. E. De Ranieri, A.W. Rushforth, K. Výborný, U. Rana, E. Ahmed, R.P. Campion, C.T. Foxon, B.L. Gallagher, A.C. Irvine, J. Wunderlich, T. Jungwirth, Lithographically and electrically controlled strain effects on anisotropic magnetoresistance in (Ga,Mn)As, New J. Phys. 10(6) (2008) 065003. 